\title{
Correction: Downregulation of microRNA-9-5p promotes synaptic remodeling in the chronic phase after traumatic brain injury
}

Jingchuan Wu (1), Hui Li, Junchi He, Xiaocui Tian, Shuilian Luo, Jiankang Li, Wei Li, Jianjun Zhong, Hongrong Zhang, Zhijian Huang, Xiaochuan Sun and Tao Jiang (D)

Correction to: Cell Death \& Disease https://doi.org/10.1038/s41419-020-03329-5 published online 5 January 2021

The original version of this article unfortunately contained a mistake in Fig. 2. The ' $F$ ' and 'G' graphs in Fig. 2 were in reverse order. The correct figure can be found below. The authors apologize for the mistake. The original article has been corrected.

Published online: 03 March 2021 


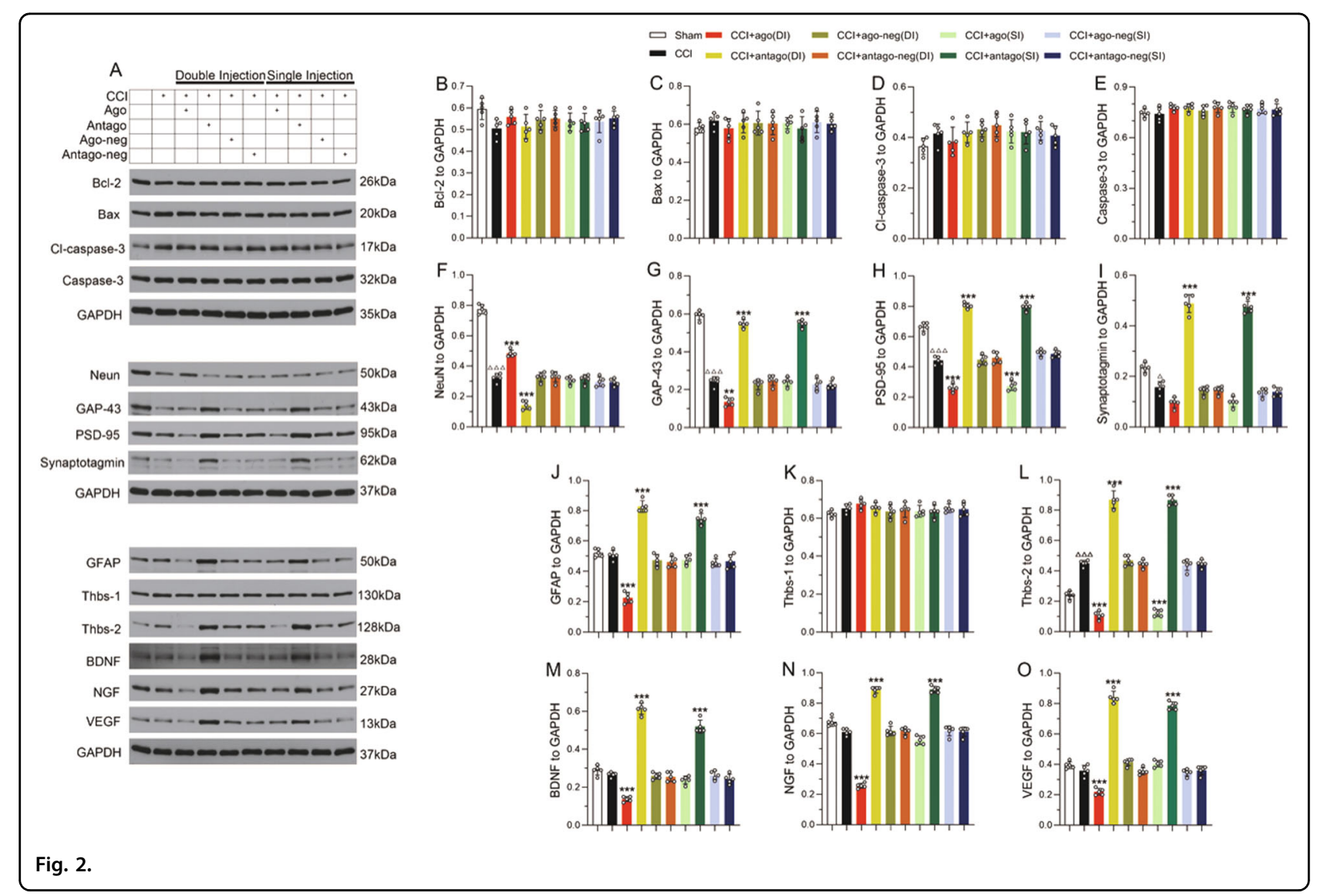

